\title{
BETTI NUMBERS OF MULTIGRADED MODULES OF GENERIC TYPE
}

\author{
HARA CHARALAMBOUS AND ALEXANDRE TCHERNEV
}

\begin{abstract}
Let $R=\mathbb{k}\left[x_{1}, \ldots, x_{m}\right]$ be the polynomial ring over a field $\mathbb{k}$ with the standard $\mathbb{Z}^{m}$-grading (multigrading), let $L$ be a Noetherian multigraded $R$-module, let $\beta_{i, \alpha}(L)$ the $i$ th (multigraded) Betti number of $L$ of multidegree $\alpha$. We introduce the notion of a generic (relative to $L$ ) multidegree, and the notion of multigraded module of generic type. When the multidegree $\alpha$ is generic (relative to $L$ ) we provide a Hochster-type formula for $\beta_{i, \alpha}(L)$ as the dimension of the reduced homology of a certain simplicial complex associated with $L$. This allows us to show that there is precisely one homological degree $i \geq 1$ in which $\beta_{i, \alpha}(L)$ is non-zero and in this homological degree the Betti number is the $\beta$-invariant of a certain minor of a matroid associated to $L$. In particular, this provides a precise combinatorial description of all multigraded Betti numbers of $L$ when it is a multigraded module of generic type.
\end{abstract}

\section{INTRODUCTION}

Throughout this paper $\mathbb{k}$ is a field, $R=\mathbb{k}\left[x_{1}, \ldots, x_{m}\right]$ is the polynomial ring over $\mathbb{k}$ with the standard $\mathbb{Z}^{m}$-grading (multigrading), $L$ is a Noetherian multigraded $R$-module with minimal multihomogeneous free presentation

$$
E \stackrel{\Phi}{\longrightarrow} G \longrightarrow L \longrightarrow 0
$$

and $S$ is a (multi)homogeneous basis of $E$. An ongoing project of the second author is to use the combinatorial properties of the free multigraded resolution $T(\Phi, S)$. of $L$ and the matroid $\mathbf{M}(\Phi, S)$ from [12] to study the homological properties of $L$. In this paper we apply this technique to investigate the multigraded Betti numbers $\beta_{i, \alpha}(L)$ in a generic situation. We introduce the notion of a multigraded module of generic type which generalizes the notion of genericity introduced previously by the authors in [7]. Our definition is new even in the case of monomial ideals, where it properly (and in a strong sense) subsumes the notion of generic ideals from [1], and differs in an essential way from the notion of genericity introduced in [10], see Examples 2.4. We also introduce the finer notion of a generic (relative to $L$ ) multidegree (the module $L$ is then of generic type if each multidegree $\alpha \in \mathbb{Z}^{m}$ is generic relative to $L)$.

When $I$ is a monomial ideal and $R / I$ is of generic type, as a first result we show that the algebraic Scarf complex is a minimal resolution of $R / I$, Corollary 2.6. Later on we show that if $I$ is a monomial ideal and $\alpha$ is a generic multidegree

1991 Mathematics Subject Classification. 13D02, 13A02, 52B40.

Key words and phrases. multigraded modules, free resolutions, matroids.

The first author is grateful to the European Union for support in the framework of the program "Pythagoras" of the "Operational Program for Education and Initial Vocational Training" of the 3rd Community Support Framework of the Hellenic Ministry of Education, for part of the project. 
then the $i$ th Betti number of $R / I$ is nonzero precisely when $\alpha$ belongs to the Scarf complex of $I$, Corollary 5.9.

The main result of this paper is the computation of the $i$ th Betti number of $L$ when $\alpha$ is generic relative to $L$, Theorem 5.8. We provide a Hochster-type formula for the multigraded Betti numbers $\beta_{i, \alpha}(L)$ in terms of the relative homology of a certain simplicial complex associated with $\alpha$ and the matroid $\mathbf{M}(\Phi, S)$. We analyze the properties of that simplicial complex to show that the Betti numbers $\beta_{i, \alpha}(L)$ are zero except in at most one degree $i$, and in that degree the Betti number equals the $\beta$-invariant of a certain minor $\mathbf{M}_{\alpha}$ of the matroid $\mathbf{M}(\Phi, S)$. In particular, this provides a detailed combinatorial description of the multigraded Betti numbers for the class of multigraded modules of generic type.

The material is organized as follows. In Section 2 we introduce the notion of a generic element in the LCM-lattice of $L$, the notion of a generic multidegree relative to $L$, and the notion of a multigraded module of generic type. We compare the new notions with the existing notions of genericity. We show that if $I$ is a generic in the sense of [1] monomial ideal then the module $R / I$ is automatically of generic type. We show that when $L$ is of generic type, then the proof of [7, Theorem 5.6] works and generalize the above theorem. In particular this implies that the algebraic Scarf complex is a minimal free resolution of $R / I$ when $R / I$ is a multigraded module of generic type.

In Section 3 we introduce the affine simplicial complexes of a matroid $\mathbf{M}$ on the set $S$. We show their homology equals the $\beta$-invariant of the matroid. When a representation $\phi$ of the matroid is given, then for any ordering $\omega$ of $S$ we introduce a certain complex of vector spaces $V(\phi, \omega)$ with the same homology.

In Section 4 when $\alpha$ is a generic element we examine certain minors $\mathbf{M}_{\alpha}$ and $\mathbf{M}^{\alpha}$ of the matroid $\mathbf{M}(\Phi, S)$ of $L$. We introduce a new complex of vector spaces $V(\alpha, \phi, \omega)$ whose homology is nonzero at precisely one position and equals the $\beta$ invariant of $\mathbf{M}_{\alpha}$.

Finally, in Section 5 we use the free multigraded resolution $T_{\bullet}(\Phi, S)$ of $[12]$ to construct a certain double complex. According to the first filtration of this complex we recover the complex $V(\alpha, \phi, \omega)$, while according to the second filtration we can compute the $\alpha$-graded Betti numbers of $L$. Thus we prove that when $\alpha$ is generic relative to $L$, the reduced homology of the affine simplicial complexes of $\mathbf{M}_{\alpha}$ determines the $\alpha$-Betti numbers of $L$. Moreover the $\alpha$-Betti number of $L$ is nonzero in exactly one position and equals the $\beta$-invariant of a minor of $\mathbf{M}_{\alpha}$.

\section{Preliminaries}

For the rest of this paper $L$ is a Noetherian multigraded $R$-module, $\Phi: E \longrightarrow G$ is a minimal free multigraded presentation of $L$, and $S$ a homogeneous basis of $E$. All vector spaces, homomorphisms and unadorned tensor operations are over $\mathbb{k}$.

1.1. Complexes of vector spaces. Let $C_{\bullet}=\left(C_{i}, \varphi_{i}\right)$ be a complex of vector spaces. The dual complex of $C$ • is the complex

$$
C_{\bullet}^{*}=\left(C_{i}^{*}, \varphi_{i}^{*}\right)=\left(\operatorname{Hom}_{\mathbb{k}}\left(C_{-i}, \mathbb{k}\right), \operatorname{Hom}_{\mathbb{k}}\left(\varphi_{-i+1}, \mathbb{k}\right)\right),
$$

its shift by an integer $k$ is the complex

$$
C[k] \bullet=\left(C[k]_{i}, \varphi[k]_{i}\right)=\left(C_{i+k},(-1)^{k} \varphi_{i+k}\right) .
$$


its shift in homological degrees by $k$ is the complex

$$
C\langle k\rangle_{\bullet}=\left(C[k]_{i}, \varphi[k]_{i}\right)=\left(C_{i+k}, \varphi_{i+k}\right),
$$

and its truncation at $k$ is the complex

$$
C_{\bullet}^{\geq k}=\left(C_{i}^{\geq k}, \varphi_{i}^{\geq k}\right)=\left(\tau_{i}^{k} C_{i}, \tau_{i}^{k} \varphi_{i}\right) \text { where } \tau_{i}^{k}= \begin{cases}\text { id } & \text { if } i \geq k ; \\ 0 & \text { otherwise. }\end{cases}
$$

We call $C_{\bullet}$ acyclic if $\mathrm{H}_{i}\left(C_{\bullet}\right)=0$ for $i \neq 0$, and exact if also $\mathrm{H}_{0}\left(C_{\bullet}\right)=0$.

1.2. Maps and vector spaces. We recall here and in the following subsections some of the notation introduced in [12]. Let $U_{S}$ be the vector space with basis the set of symbols $\left\{e_{a} \mid a \in S\right\}$. For each subset $A \subseteq S$ we denote by $U_{A}$ the subspace of $U_{S}$ spanned by the set $\left\{e_{a} \mid a \in A\right\}$. Whenever a map of vector spaces $\phi: U_{S} \longrightarrow W$ is given and $A \subseteq S$ we denote by $V_{A}$ the subspace of $W$ spanned by the set $\left\{\phi\left(e_{a}\right) \mid a \in A\right\}$; thus $V_{A}=\phi\left(U_{A}\right)$. We denote by $\phi_{A}: U_{A} \longrightarrow W$ the restriction of $\phi$ to $U_{A}$.

Next, we consider $\mathbb{k}$ as an $R$-module via the canonical $\mathbb{k}$-algebra map $R \longrightarrow \mathbb{k}$ that sends each variable $x_{i}$ to $1 \in \mathbb{k}$. We denote by $W(\Phi)$ the $\mathbb{k}$-vector space $G \otimes_{R} \mathbb{k}$. Since the set $\{a \otimes 1 \mid a \in S\}$ forms a basis of the space $E \otimes_{R} \mathbb{k}$ we can canonically identify it with $U_{S}$ by identifying $e_{a}$ with $a \otimes 1$ for each $a \in S$. We denote by $\phi(\Phi)$ the $\mathbb{k}$-linear map $\Phi \otimes_{R} \mathbb{k}$; thus we have $\phi(\Phi): U_{S} \longrightarrow W(\Phi)$.

For $A \subset S$ we let $E_{A}$ denote the free direct summand of $E$ generated over $R$ by the set $\{a \mid a \in A\}$, and let $\Phi_{A}$ denote the restriction of $\Phi$ to $E_{A}$. It is straightforward that $\phi\left(\Phi_{A}\right)=\phi(\Phi)_{A}$.

1.3. Matroids. For more details on the basic properties of matroids we refer the reader to $[14,11]$. For a quick summary with notation in the spirit of this paper see [12, Section 1].

Let $P(S)$ be the collection of all subsets of $S$, partially ordered by inclusion. Recall that any matroid $\mathbf{M}$ on $S$ is determined by a nonempty set $\mathcal{I}(\mathbf{M}) \subset P(S)$ (or $\mathcal{I}$ if clear from the context) whose elements are called the independent sets of M. The set $\mathcal{I}$ has the following three properties:

- $\emptyset \in \mathcal{I}$;

- if $I \subset J$ and $J \in \mathcal{I}$ then $I \in \mathcal{I}$;

- if $J \in P(S)$ and $I_{1}, I_{2}$ are two subsets of $J$ maximal with respect to membership in $\mathcal{I}$ then $\left|I_{1}\right|=\left|I_{2}\right|$. (This common size is called the rank of $J$ in $\mathbf{M}$ and is denoted by $r(J))$.

When a map of vector spaces $\phi: U_{S} \longrightarrow W$ is given, one obtains a matroid $\mathbf{M}(\phi)$ on $S$ by letting the set $\mathcal{I}$ consist of all subsets $I$ of $S$ for which $|I|=\operatorname{dim}_{\mathbb{k}} V_{I}$. In this case for any subset $J$ of $S$ we let $r(J)=\operatorname{dim}_{\mathbb{k}} V_{J}$. One says that $\mathbf{M}(\phi)$ is represented by $\phi$ over $\mathbb{k}$ and that $\phi$ is a representation over $\mathbb{k}$ of $\mathbf{M}(\phi)$. In the case at hand where $\Phi: E \longrightarrow G$ is a minimal free multigraded presentation of $L$, we consider the matroid $\mathbf{M}(\Phi, S):=\mathbf{M}(\phi(\Phi))$.

Recall that a circuit of a matroid $\mathbf{M}$ is a minimal dependent set, and a loop of $\mathbf{M}$ is an element $a \in S$ so that $\{a\}$ is a circuit. A $T$-flat is a subset of $S$ that is a union of circuits. A flat of $\mathbf{M}$ is subset $B \subset S$ such that $r(B \cup c)=r(B)+1$ for each $c \notin B$. A hyperplane of $\mathbf{M}$ is a maximal proper flat, i.e. a flat $H$ such that $r(H)=r(S)-1$. The collection of T-flats forms a lattice with respect to inclusion. The intersection of flats is a flat. The matroid closure $\bar{B}$ of a subset $B \subseteq S$ is the 
smallest flat containing $B$ : it equals the intersection of all flats containing $B$. Two elements $a, b \in S$ are called parallel if they are not loops and $\overline{\{a\}}=\overline{\{b\}}$. Let $J \subset S$. The restriction of $\mathbf{M}$ to $J$ is the matroid $\mathbf{M} \mid J$ whose independent sets form the set $\mathcal{I}(\mathbf{M} \mid J)=\mathcal{I}(\mathbf{M}) \cap P(J)$. The contraction of $\mathbf{M}$ to $J$ is the matroid $\mathbf{M} . J$ whose independent sets form the set $\mathcal{I}(\mathbf{M} . J)=\left\{I \subset J \mid I \cup I^{\prime} \in \mathcal{I}(\mathbf{M}), \forall I^{\prime} \in \mathcal{I}(\mathbf{M} \mid S \backslash J)\right\}$. The $\beta$-invariant of $\mathbf{M}$ is

$$
\beta(\mathbf{M})=(-1)^{r(S)} \sum_{J \subset S}(-1)^{|J|} r(J) .
$$

One of the important properties of the $\beta$-invariant is that $\beta(\mathbf{M})=0$ is zero whenever $\mathbf{M}$ has a loop, see [8, Theorem II].

1.4. Multigraded resolutions. For the rest of the paper we denote by $T_{\bullet}(\Phi, S)$ the free multigraded resolution of $L$ from [12, Theorem 4.5]. In homological degrees 0 and 1 the resolution $T_{\bullet}(\Phi, S)$ is simply the minimal presentation $\Phi$. For $n \geq 2$, the $R$-components of $T_{\bullet}(\Phi, S)$ are:

$$
T_{n}(\Phi, S)=\bigoplus_{I}\left(T_{I} \otimes R\right)[-\operatorname{deg} I]
$$

where the index $I \subset S$ runs through all T-flats of $\mathbf{M}$ such that $r(I)=|I|-n+1$, $\operatorname{deg} I$ is the componentwise maximum of the multidegrees of the elements of $I, T_{I}$ is a certain $\mathbb{k}$-vector space associated to $I$, see [12, Definition 2.2.3], and $\left(T_{I} \otimes R\right)[-\operatorname{deg} I]$ is the free module $T_{I} \otimes R$ shifted by multidegree $\operatorname{deg} I:\left(\left(T_{I} \otimes R\right)[-\operatorname{deg} I]\right)_{\alpha}=\left(T_{I} \otimes\right.$ $R)_{\alpha+\operatorname{deg} I}$ for any $a \in \mathbb{Z}^{m}$. While in general the resolution $T_{\bullet}(\Phi, S)$ is not minimal, we will use it to obtain information about the minimal multigraded resolution of $L$. We denote by $\beta_{i, \alpha}(L)$ the $i$ th multigraded Betti number of $L$ :

$$
\beta_{i, \alpha}(L)=\operatorname{dim}_{\mathbb{k}} \mathrm{H}_{i}(T(\Phi, S) \otimes \mathbb{k})_{\alpha}=\operatorname{dim}_{\mathbb{k}} \operatorname{Tor}_{i}^{R}(L, \mathbb{k})_{\alpha} .
$$

\section{Multigraded modules of Generic type}

First we recall the definition of $L C M$-lattice of the multigraded module $L$.

Definition 2.1. Let $\Lambda=\Lambda(L)$ be the lattice in $\mathbb{Z}^{m}$ (with the join operation being componentwise maximum) join-generated by the multidegrees of the elements of $S$. We call $\Lambda$ the $L C M$-lattice of $L$. Since the collection of multidegrees $\{\operatorname{deg} a \mid a \in S\}$ of the elements of $S$ is independent of the choice of the basis $S$, the LCM-lattice is an invariant of $L$.

Remark 2.2. The multidegrees of the free modules in $T_{\bullet}(\Phi, S)$ are elements of the LCM-lattice $\Lambda(L)$. It follows that the minimal syzygies of $L$ can occur only in multidegrees $\alpha$ that belong to $\Lambda(L)$. Consequently, for $i \geq 1$ the Betti numbers $\beta_{i, \alpha}(L)$ can be nonzero only if $\alpha \in \Lambda(L)$.

Consider the degree map of posets

$$
\operatorname{deg}: P(S) \longrightarrow \mathbb{Z}^{m}
$$

given by $\operatorname{deg} A=\bigvee\{\operatorname{deg} a \mid a \in A\}$ for each subset $A \subseteq S$, and note that $\Lambda(L)$ is precisely the image of the map deg. For each $\alpha \in \Lambda(L)$ there always is a unique maximal set $I^{\alpha}$ in $P(S)$ of degree $\alpha$ : $I^{\alpha}$ equals the union of all sets of degree $\leq \alpha$. 
Definition 2.3. We say that $\alpha \in \Lambda(L)$ is a generic element of $\Lambda(L)$ if the fiber $\operatorname{deg}^{-1}(\alpha)$ is a closed interval in $P(S)$, i.e. if there is a unique minimal subset of $S$ of degree $\alpha$ denoted by $I_{\alpha}$ and $\operatorname{deg}^{-1}(\alpha)=\left[I_{\alpha}, I^{\alpha}\right]$.

We say that $\alpha \in \mathbb{Z}^{m}$ is generic relative to $L$ if either $\alpha \notin \Lambda(L)$ or if $\alpha$ is a generic element of $\Lambda(L)$.

We say that $\Lambda(L)$ is of generic type if each $\alpha \in \mathbb{Z}^{n}$ is generic relative to $L$ and in this case we say that $L$ is also of generic type.

It is immediate that if $L$ is of generic type then no two elements of $S$ have the same multidegree. The notion of generic type only depends on the multidegrees of the basis elements of $S$ and is independent of the choice of the particular basis $S$. Below we give some examples to differentiate between the different notions of generic.

\section{Examples 2.4.}

- The Scarf simplicial complex of $\Phi, \Delta(\Phi)$ is the subcomplex of $P(S)$ consisting of all subsets $I$ so that $\operatorname{deg}^{-1}(\operatorname{deg} I)=\{I\}$.

- Let $J=\left(x^{2}, x y, x z\right)$. Then $R / J$ is of generic type as can be readily checked, and $J$ is not generic in the sense of [1] or [10].

- If $J$ is a monomial ideal generic in the sense of [1], then $R / J$ is of generic type. Indeed, for $\alpha \in \Lambda(R / J)$ take $s_{i} \in S$ to be the unique monomial generator of $I$ that agrees with $\alpha$ in the $i$ th coordinate. The unique minimal set $I_{\alpha}$ of degree $\alpha$ is the collection of the distinct $s_{i}$ obtained this way.

- Let $J=\left(x^{3} z^{2}, x^{2} y^{3}, x y^{2} z, y^{3} z^{2}\right)$. Then $(3,3,2)$ is not a generic element of $\Lambda(R / J)$ as $\{1,2\},\{1,4\}$ are minimal in $\operatorname{deg}^{-1}(3,3,2)$. We note that $J$ is generic in the sense of [10].

- Let $I$ be a monomial ideal, $L=R / I$, and $\Delta_{L}$ be the Scarf complex of $L$, see [1] or [10]. If $\sigma \in \Delta_{L}$ then the multidegree $\alpha$ of $\sigma$ is a generic element of $\Lambda(L)$ and $\operatorname{deg}^{-1}(\alpha)=I^{\alpha}=I_{\alpha}$ is just a point.

- Let $I$ be a monomial ideal in $R$ and $J$ the polarization of $I$ in a polynomial ring $S$. It is clear that $R / I$ is of generic type if and only if $S / J$ is of generic type. This is not the case when $I$ is generic in the sense of [10] as the simple example $\left(x^{2}, x y\right)$ demonstrates.

- If $I$ is generic in the sense of [10] then $I^{*}=I+\left(x_{1}^{D}, \ldots, x_{m}^{D}\right)$ (where $D$ is sufficiently large) is also generic in the sense of [10]. Let $I=(x y, x z)$. $R / I$ is of generic type and as we will see below the algebraic Scarf complex is a minimal free resolution of $R / I$. However for $D>1$ the ideal $I^{*}=$ $I+\left(x^{D}, y^{D}, z^{D}\right)$ is not of generic type since $\operatorname{deg}^{-1}((1, D, D))$ is not an interval.

Let $r=\operatorname{rank} \Phi, g=\operatorname{rank}_{R}(G)$. We recall from [7] that $\Phi$ is of uniform rank if all $g \times r$ submatrices of the coefficient matrix of $\Phi$ have rank equal to $r$. In [7] the Scarf complex of $\Phi, S_{\bullet}(\Phi)$ was introduced. When $g=1, S_{\bullet}(\Phi)$ is the algebraic Scarf complex, $F_{\Delta(\Phi)}$, of [1]. In [7, Theorem 5.6] it is shown that when $\Phi$ is of uniform rank and $L$ is generic in the sense of [1] then $S_{\bullet}(\Phi)$ is a minimal free multigraded resolution of $L$. The condition needed for the proof of [7, Theorem 5.6] to work is that there is a unique minimal face of degree $\alpha$, so that $i \in I^{\alpha} \backslash I_{\alpha}$ if and only if $\operatorname{deg}\left(I^{\alpha} \backslash i\right)=\operatorname{deg}\left(I^{\alpha}\right)=\alpha,[7, \operatorname{pg} 547]$. This condition is is equivalent to $\alpha$ being a generic element of $\Lambda(L)$. Thus the next theorem holds: 
Theorem 2.5. Let $\Phi: E \longrightarrow G$ be a minimal free multigraded presentation of the multigraded module $L$ so that $\Phi$ is of uniform rank and $L$ is of generic type. Then $S \bullet(\Phi)$ is a minimal free multigraded resolution of $L$.

We apply the above when $R / I$ is of generic type:

Corollary 2.6. Let $I$ be a monomial ideal so that $R / I$ is of generic type and $\Phi: R^{n} \longrightarrow R$ a minimal multigraded presentation of $R / I$. The algebraic Scarf complex $F_{\Delta(\Phi)}$ is a minimal free multigraded resolution of $R / I$.

Proof. Minimality of the presentation $\Phi$ implies that $\Phi$ is of uniform rank.

Let $I$ be a monomial ideal and let $\Phi^{*}$ be the minimal free multigraded presentation of $R / I^{*}$. According to [10], $I$ is generic if and only if $F_{\Delta\left(\Phi^{*}\right)}$ is a minimal free resolution of $R / I^{*}$. Thus if $I$ is not generic according to [10] but $R / I$ is of generic type then $F_{\Delta\left(\Phi^{*}\right)}$ is not acyclic and $R / I^{*}$ is not of generic type.

\section{Affine Simplicial COMPleXes and the $\beta$-InVARiant}

Let $\mathbf{M}$ be an arbitrary matroid on the set $S, \phi: U_{S} \longrightarrow W$ a representation of $\mathbf{M}$ and $\left\{e_{a} \mid a \in S\right\}$ a basis of $U_{S}$. For any $b \in S$ we introduce a simplicial complex and compute its homology.

Definition 3.1. Let $b \in S$. We let $\Delta_{b}$ be the simplicial complex

$$
\Delta_{b}=\{J \subset P(S) \mid b \notin \bar{J}\}
$$

We call $\Delta_{b}$ the affine simplicial complex of $\mathbf{M}$ away from $b$.

We note that the facets of $\Delta_{b}$ are the hyperplanes of the matroid $\mathbf{M}$ that do not contain $b$ and that $J \in \Delta_{b}$ if and only if $V_{J} \neq V_{J \cup b}$.

Theorem 3.2. Let $b \in S$. Then for $i \geq 0$

$$
\operatorname{dim}_{\mathbb{k}} \widetilde{\mathrm{H}}_{i}\left(\Delta_{b}, \mathbb{k}\right)= \begin{cases}\beta(\mathbf{M}) & \text { if } i=r(\mathbf{M})-2 \\ 0 & \text { otherwise. }\end{cases}
$$

Furthermore, if $b$ is not a loop then the above equality is true for all $i$.

Proof. If $\mathbf{M}$ has a loop $c$ then $\beta(\mathbf{M})=0$. Furthermore, in that case $\Delta_{b}$ is either the empty simplicial complex (when $b$ is a loop) or a cone with apex $c$. Thus in the sequel we assume that $\mathbf{M}$ has no loops.

Next we define a new complex $\bar{\Delta}_{b}$ as follows: the set of vertices of $\bar{\Delta}_{b}$ is

$$
\operatorname{Vert}\left(\bar{\Delta}_{b}\right)=\{J \mid J \text { is a flat in } \mathbf{M} \text { of rank } 1 \text { such that } b \notin J\} \text {. }
$$

The faces of $\bar{\Delta}_{b}$ are the sets

$$
\left\{J_{1}, \ldots, J_{t}\right\}: J_{1} \cup \cdots \cup J_{t} \subset H \text {, for some facet } H \text { of } \Delta_{b} \text {. }
$$

Since $\mathbf{M}$ has no loops we can define the simplicial map $\pi: \Delta_{b} \longrightarrow \bar{\Delta}_{b}$ that sends each vertex $y$ of $\Delta_{b}$ to its matroid closure $\overline{\{y\}}$, which is a flat of rank 1 . Since a facet of $\Delta_{b}$ contains $y$ if and only if it contains $\overline{\{y\}}$, it is straightforward that $\pi$ is a quotient map arising from partitioning the vertices of $\Delta_{b}$ into classes of parallel elements. Therefore the Contractible Subcomplex Lemma [3, (2.2)] yields that $\pi$ is a homotopy equivalence. Let $L_{b}$ be the poset obtained by removing from the lattice of flats of $\mathbf{M}$ those flats that contain $b$. Then by the Crosscut Theorem [2, Theorem 2.3], $\bar{\Delta}_{b}$ is homotopy equivalent with the order complex of the poset 
$L_{b}^{o}$ obtained by removing from $L_{b}$ its minimal element. By the results of Wachs and Walker [13, Theorem 3.2 and Corollary 7.2] this order complex is a shellable simplicial complex, and its reduced homology has already been computed, see e.g. [3] and [15, Theorem 2.6] or [5, Theorem 3.12]. In particular, it is possibly nonzero only in dimension $r(\mathbf{M})-2$, and its rank there is precisely the $\beta$-invariant of $\mathbf{M}$.

Next we compare the homology of $\Delta_{b}$ with the homology of a complex of vector spaces determined by the subspaces of $V_{S}$. Let $\omega$ be an ordering on $S$ and we use this ordering to identify each subset of $S$ with the increasing sequence of its elements. For each $J \subset S$ and $c \notin J$, we have that $V_{J}$ is a subset of $V_{J \cup\{c\}}$. We let

$$
V(\phi, \omega)_{i}=\bigoplus_{B \subset S,|B|=|S|-i} V_{B}
$$

and $V(\phi, \omega)$. be the complex

$$
0 \longrightarrow \bigoplus_{B \subset S,|B|=1} V_{B} \longrightarrow \ldots \longrightarrow \bigoplus_{B \subset S,|B|=|S|-1} V_{B} \longrightarrow V_{S} \longrightarrow 0
$$

where at the $i$ th stage the maps componentwise are the inclusions $V_{B} \rightarrow V_{B \cup\{c\}}$ times the sign of the permutation that arranges the sequence $(c, B)$ in increasing order then followed by composition with the natural inclusion $V_{B \cup\{c\}} \hookrightarrow V(\phi, \omega)_{i+1}$.

Lemma 3.4. If $b \in S$ is not a loop of $\mathbf{M}$ then

$$
\mathrm{H}_{i}(V(\phi, \omega) \bullet) \cong \widetilde{\mathrm{H}}_{|S|-i-2}\left(\Delta_{b}, \mathbb{k}\right)
$$

for all $i$.

Proof. Let $\widetilde{Y}$ be the reduced chain complex of $\Delta_{b}$ over $\mathbb{k}$. Since the complexes $V(\phi, \omega)$. are canonically isomorphic for different choices of $\omega$, we may assume that $b$ is the greatest element of $S$ and that $\omega$ induces the orientation on the faces of $\Delta_{b}$ used to construct $\widetilde{Y}$. We write $V_{\bullet}$ for $V(\phi, \omega)$. We consider a certain subcomplex $D_{\bullet}$ of $V_{\bullet}$. We let $D_{i}=\bigoplus_{J} V_{J}$ where $b \in J$ and $|J|=|S|-i$. In particular $D_{0}=V_{S}$ and

$$
D_{\bullet}: 0 \rightarrow V_{b} \longrightarrow \cdots \longrightarrow V_{S} \rightarrow 0
$$

where $D_{i} \rightarrow D_{i-1}$ are the restrictions of the maps from (3.3). Let

$$
E_{\bullet}=\left(V_{\bullet} / D_{\bullet}\right)[1] .
$$

If $b \notin J$, we let $\psi_{J}$ be the inclusion $V_{J} \subset V_{J \cup b}$ taken with the sign $(-1)^{|J|}$. Then $\psi: E_{\bullet} \longrightarrow D_{\bullet}$. defined componentwise by the maps $\psi_{J}$ is an injective map of complexes and the complex $V_{\bullet}$ is precisely the mapping cone of $\psi$. We let

$$
\bar{D}_{\bullet}=D_{\bullet} / \psi\left(E_{\bullet}\right) \text {. }
$$

It follows from the injectivity of $\psi$ and the standard properties of mapping cones that $\mathrm{H}_{i}\left(V_{\bullet}\right) \cong \mathrm{H}_{i}\left(\bar{D}_{\bullet}\right)$ for each $i$. Next we note that the nonzero summands of $\bar{D}_{i}$ are of the form $V_{J \cup b} / V_{J}$ where $V_{J} \neq V_{J \cup b}$ and $|J|=|S|-1-i$. Thus the sets $J$ involved are precisely the faces of $\Delta_{b}$. We consider $Y^{\prime}=\widetilde{Y}^{*}\langle-|S|+2\rangle$, (so that that $\operatorname{Hom}_{\mathbb{k}}\left(\widetilde{Y}_{-1}, \mathbb{k}\right)$ is in homological degree $\left.|S|-1\right)$. For $J \in \Delta_{b}$ we let $\sigma_{J}^{*}$ be the standard generator of $Y^{\prime}$ associated to $J$. Identifying $\sigma_{J}^{*}$ with $\phi\left(e_{b}\right)+V_{J}$ in $V_{J \cup b} / V_{J}$ we see that $Y^{*}\langle-|S|+2\rangle$ can be identified with $\bar{D}$. and the lemma follows.

We are now ready to compute the homology of the complex $V(\phi, \omega)$ : 


\section{Theorem 3.5.}

$$
\operatorname{dim}_{\mathbb{k}} \mathrm{H}_{i}\left(V(\phi, \omega) \bullet= \begin{cases}\beta(\mathbf{M}) & \text { if } i=|S|-r(\mathbf{M}) \\ 0 & \text { otherwise. }\end{cases}\right.
$$

Proof. If $\mathbf{M}$ has an element $b$ that is not a loop, then the theorem is immediate by combining Theorem 3.2 and Lemma 3.4. If all elements of $S$ are loops in $\mathbf{M}$ then $r(\mathbf{M})=0$, the complex $V(\phi, \omega)$. is zero, and the desired conclusion is immediate from the fact that $\beta(\mathbf{M})=0$.

\section{Minors of $\mathbf{M}(\Phi, S)$ associated with a Generic multidegree}

Let $\alpha \in \Lambda(L)$ be a generic element. For the rest of this paper we fix $\mathbf{M}=$ $\mathbf{M}(\Phi, S)$, we set $W=W(\Phi)$, and $\phi=\phi(\Phi): U_{S} \longrightarrow W$ (see Section 1). We introduce some new matroids associated with $\alpha$. Recall that $\operatorname{deg}^{-1}(\alpha)=\left[I_{\alpha}, I^{\alpha}\right]$ is a closed interval in the boolean poset $P(S)$.

\section{Definition 4.1.}

- We set $I(\alpha)=I^{\alpha} \backslash I_{\alpha}$.

- We denote by $\mathbf{M}^{\alpha}$ the matroid that is the restriction of $\mathbf{M}$ to $I^{\alpha}$. In standard matroid notation we have $\mathbf{M}^{\alpha}=\mathbf{M} \mid I^{\alpha}$.

- We denote by $\mathbf{M}_{\alpha}$ the matroid that is the contraction of $\mathbf{M}^{\alpha}$ to $I_{\alpha}$. In standard matroid notation we have $\mathbf{M}_{\alpha}=\mathbf{M}^{\alpha} \cdot I_{\alpha}=\mathbf{M}^{\alpha} / I(\alpha)$.

We discuss the above matroids in terms of some linear transformations associated with $\alpha$.

\section{Remarks 4.2.}

- $\mathbf{M}^{\alpha}$ is represented by $\phi_{I^{\alpha}}: U_{I^{\alpha}} \rightarrow W$ over $\mathbb{k}$.

- Let $\pi_{\alpha}: W \rightarrow W / V_{I(\alpha)}$ be the canonical projection map, and let

$$
\overline{\phi_{I_{\alpha}}}:=\pi_{\alpha} \circ \phi_{I_{\alpha}}: U_{I_{\alpha}} \rightarrow W / V_{I(\alpha)} .
$$

The matroid $\mathbf{M}_{\alpha}$ is represented by $\overline{\phi_{I_{\alpha}}}$ over $\mathbb{k}$.

- We set $\bar{V}_{I_{\alpha}}:=V_{I^{\alpha}} / V_{I(\alpha)}$. For each $B \subset I_{\alpha}, V_{I(\alpha)}$ is a subspace of $V_{I(a) \cup B}$ and we set $\bar{V}_{B}:=V_{I(a) \cup B} / V_{I(\alpha)}=\overline{\phi_{I_{\alpha}}}\left(U_{B}\right)$.

- Let $\omega$ be an ordering on $I_{\alpha}$. According to the definition

$$
V\left(\overline{\phi_{I_{\alpha}}}, \omega\right) \bullet: \quad 0 \rightarrow \bigoplus_{B \subset I_{\alpha},|B|=1} \bar{V}_{B} \rightarrow \ldots \rightarrow \bar{V}_{I_{\alpha}} \rightarrow 0 .
$$

Next we define a new complex with the same homology as $V\left(\overline{\phi_{I_{\alpha}}}, \omega\right)$.

Definition 4.4. Let $\omega$ be an ordering on $I_{\alpha}$. We let

$$
V(\alpha, \phi, \omega)_{i}=\bigoplus_{\substack{A \subset I_{\alpha} \\|A|=i}} V_{I^{\alpha} \backslash A}
$$

and define a complex of vector spaces $V(\alpha, \phi, \omega) \bullet$ as the sequence

$$
0 \rightarrow V_{I(\alpha)} \rightarrow \ldots \rightarrow \bigoplus_{\substack{A \subset I_{\alpha} \\|A|=1}} V_{I^{\alpha} \backslash A} \rightarrow V_{I^{\alpha}} \rightarrow 0
$$




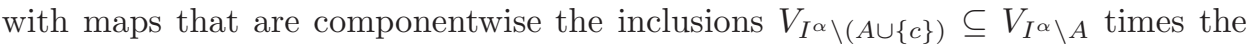
sign of the permutation that arranges the sequence $\left(c, I_{\alpha} \backslash A\right)$ in increasing order then followed by composition with the natural inclusion.

Let $\widetilde{C}$ be the reduced chain complex over $\mathbb{k}$ for the full simplex on the set $I_{\alpha}$ as oriented by $\omega$. Let $C^{\prime}=(\widetilde{C})^{*}\left\langle-\left|I_{\alpha}\right|+1\right\rangle$, (so that $\operatorname{Hom}_{\mathbb{k}}\left(\widetilde{C}_{-1}, \mathbb{k}\right)$ is in homological degree $\left.\left|I_{\alpha}\right|\right)$. We will consider the complex $V_{I(\alpha)} \otimes C^{\prime}$. Let $\sigma_{A}^{*}$ be the standard generator of $C^{\prime}$ associated to $A \subset I_{\alpha}$. We identify $V_{I(\alpha)} \otimes \sigma_{A}^{*}$ with the subspace $V_{I(\alpha)}$ of $V_{I(\alpha) \cup B}$ via the map $v \otimes \sigma_{A}^{*} \mapsto v$, where $B=I_{\alpha} \backslash A$. With this identification it is easy to see that the following holds:

Proposition 4.6. The complex $V_{I(\alpha)} \otimes C^{\prime}$ is a subcomplex of $V(\alpha, \phi, \omega)$. and we have the short exact sequence of complexes

$$
0 \rightarrow V_{I(\alpha)} \otimes C^{\prime} \rightarrow V(\alpha, \phi, \omega) \bullet V\left(\overline{\phi_{I_{\alpha}}}, \omega\right) \bullet 0 .
$$

We note that $V_{I(\alpha)} \otimes C^{\prime}$ is an exact complex. Thus taking the long exact sequence on the homology of (4.7) yields

Lemma 4.8. $\mathrm{H}_{i}(V(\alpha, \phi, \omega) \bullet)=\mathrm{H}_{i}\left(V\left(\overline{\phi_{I_{\alpha}}}, \omega\right) \bullet\right)$.

Combining Theorem 3.5 and Lemma 4.8 we obtain the following

Corollary 4.9. Let $\omega$ be an ordering on $I_{\alpha}$. Then for all $i$ we have

$$
\operatorname{dim}_{\mathbb{k}} \mathrm{H}_{i}(V(\alpha, \phi, \omega) \bullet)= \begin{cases}\beta\left(\mathbf{M}_{\alpha}\right) & \text { if } i=\left|I_{\alpha}\right|-r\left(\mathbf{M}_{\alpha}\right) \\ 0 & \text { otherwise. }\end{cases}
$$

We finish this section with an example to demonstrate the above.

Example 4.10. Let $R=\mathbb{Q}[x, y, z], E \cong R^{4}$ a multigraded free module with basis $S=\{a, b, c, d\}$ where $\operatorname{deg} a=(3,1,1), \operatorname{deg} b=(1,3,1), \operatorname{deg} c=(1,1,3), \operatorname{deg} d=$ $(1,2,2), G \cong R^{2}$ and $L$ multigraded with minimal multigraded free presentation

$$
E \stackrel{\Phi}{\longrightarrow} G \longrightarrow L \longrightarrow 0
$$

and such that the matrix of $\phi(\Phi)$ according to the bases $\{e \otimes 1 \mid e \in S\}$ and the canonical basis of $G$ is given by the matrix

$$
\left[\begin{array}{llll}
1 & 1 & 1 & 1 \\
1 & 1 & 2 & 3
\end{array}\right] .
$$

First we examine the case where $\alpha=(3,3,3)$. Here $I^{\alpha}=\{a, b, c, d\}, I_{\alpha}=\{a, b, c\}$ and $I(\alpha)=\{d\}$. Thus $\beta\left(\mathbf{M}_{\alpha}\right)=1, r\left(M_{a}\right)=1$ and for any ordering $\omega$ on $I_{\alpha}$ the homology of the complex

$$
V(\alpha, \phi, \omega): \quad 0 \rightarrow \mathbb{Q} \longrightarrow \mathbb{Q}^{6} \longrightarrow \mathbb{Q}^{6} \longrightarrow \mathbb{Q}^{2} \rightarrow 0
$$

is nonzero precisely for $i=2$.

When $\alpha=(3,2,3), \operatorname{deg}^{-1}(\alpha)$ equals the point $\{a, c, d\}$, while $r\left(M_{a}\right)=2$ and $\beta\left(M_{\alpha}\right)=1$. For any ordering $\omega$ on $I_{\alpha}$ the homology of the complex

$$
V(\alpha, \phi, \omega): \quad 0 \rightarrow \mathbb{Q}^{3} \longrightarrow \mathbb{Q}^{6} \longrightarrow \mathbb{Q}^{2} \rightarrow 0
$$

is nonzero precisely for $i=1$. 


\section{The Betti numbers of $L$}

Let $T_{\bullet}(\Phi, S)$ be the multigraded free resolution of $L$, see Section 1.4 and let $\alpha \in \Lambda(L)$. We examine the $\alpha$-graded piece of $T_{n}(\Phi, S)$ for $n \geq 2$ in order to compute $\beta_{i, \alpha}(L)$. We have that

$$
T_{n}(\Phi, S)_{\alpha}=\bigoplus_{J, \operatorname{deg} J+\beta=\alpha}\left(T_{J} \otimes R\right)[-\operatorname{deg} J]_{\beta}
$$

where the index $J \subset S$ runs through all T-flats of $\mathbf{M}$ such that $r(J)=|J|-n+1$. It follows that $\operatorname{deg} J \leq \alpha$ and that $J \subset I^{\alpha}$. Let $m$ be the maximal multigraded ideal of $R$. It is clear that

$$
m T_{i}(\Phi, S) \cap T_{i}(\Phi, S)_{\alpha}=\bigoplus_{\substack{J, \operatorname{deg} J+\beta=\alpha \\ \operatorname{deg} J<\alpha}}\left(T_{J} \otimes R\right)[-\operatorname{deg} J]_{\beta} .
$$

When $\alpha$ is a generic element the condition $\operatorname{deg} J<\alpha$ is equivalent to the existence of an element $b \in I_{\alpha}$ such that $J \subset I^{\alpha} \backslash\{b\}$.

Let $A \subset S$. We let

$$
\mathbf{T}_{\bullet}\left(\phi_{A}\right)=T_{\bullet}\left(\Phi_{A}, A\right)^{\geq 1}\langle 1\rangle \otimes_{R} \mathbb{k} .
$$

The complex $\mathbf{T} \bullet\left(\phi_{A}\right)$ was introduced in [12, Definition 2.4.1] where it was shown that

$$
\mathbf{T}_{\bullet}\left(\phi_{A}\right) \rightarrow V_{A} \rightarrow 0
$$

is exact. We note that $\mathbf{T}_{0}\left(\phi_{A}\right)=U_{A}$. We will need the following important property, see [12, Theorem 3.2(b) and Theorem 3.5]: if $A \subset B$ then $\mathbf{T}_{\bullet}\left(\phi_{A}\right)$ is canonically a subcomplex of $\mathbf{T}_{\bullet}\left(\phi_{B}\right)$. The following Lemma is a straightforward consequence of the basic structure of these complexes.

Lemma 5.1. Let $\omega$ be an ordering in $Y \subset S$ and $X_{i}$ a collection of subsets of $Y$. There is a chain map $p: \bigoplus \mathbf{T}\left(\phi_{X_{i}}\right) \rightarrow \mathbf{T}\left(\phi_{Y}\right)$ where $\left.p\right|_{\mathbf{T}\left(\phi_{X_{i}}\right)}$ equals the canonical inclusion map times the sign determined by $\omega$ to order the elements of $\left(Y \backslash X_{i}, Y\right)$.

Let $\alpha \in \Lambda(L)$ be a generic element. Fix an ordering $\omega$ in $I^{\alpha}$ and consider the chain map

$$
p: \bigoplus_{b \in I_{\alpha}} \mathbf{T}\left(\phi_{I^{\alpha} \backslash\{b\}}\right) \rightarrow \mathbf{T}\left(\phi_{I^{\alpha}}\right)
$$

as in Lemma 5.1. We introduce a new complex:

Definition 5.2. Let $C(\alpha)$ • be the following complex of vector spaces:

$$
C(\alpha) \cdot=\mathbf{T}\left(\phi_{I^{\alpha}}\right) / p\left(\bigoplus_{b \in I_{\alpha}} \mathbf{T}\left(\phi_{I^{\alpha} \backslash\{b\}}\right) .\right.
$$

We note that for $i \geq 1$ we have

$$
m T_{i}(\Phi, S) \cap T_{i}(\Phi, S)_{\alpha}=p\left(\bigoplus_{b \in I_{\alpha}} \mathbf{T}\left(\phi_{I^{\alpha} \backslash\{b\}}\right)\right)_{i-1}
$$

and thus the following lemma holds:

Lemma 5.3. Let $\alpha \in \Lambda$ be a generic element. Then for $i \geq 1$

$$
\beta_{i, \alpha}(L)=\operatorname{dim}_{\mathbb{k}} \mathrm{H}_{i-1}(C(\alpha) \bullet) .
$$


Proof. Since $\beta_{i, \alpha}(L)=H_{i}\left(T(\Phi, S) \otimes_{R} \mathbb{k}\right)_{\alpha}$, we combine the above remarks to get

$$
\left(T_{i}(\Phi, S) \otimes_{R} \mathbb{k}\right)_{\alpha}=T_{i}(\Phi, S)_{\alpha} / m T_{i}(\Phi, S) \cap T_{i}(\Phi, S)_{\alpha}=C_{i-1}(\alpha) .
$$

In view of the minimality of the presentation $\Phi$, the Lemma is now immediate.

We will reduce the study of the homology of $C(\alpha)$ • to the study of a certain double complex. We will need the following lemma:

Lemma 5.4. Let $X \subset Y \subset S$, and $\omega$ be a linear ordering $Y$. Consider the sequence

$$
\begin{aligned}
\mathbf{T}(X, Y, \omega): 0 & \rightarrow \mathbf{T}\left(\phi_{X}\right) \longrightarrow \bigoplus_{b \in Y \backslash X} \mathbf{T}\left(\phi_{X \cup\{b\}}\right) \longrightarrow \bigoplus_{b, c \in Y \backslash X} \mathbf{T}\left(\phi_{X \cup\{b, c\}}\right) \\
& \longrightarrow \ldots \longrightarrow \bigoplus_{c \in Y \backslash X} \mathbf{T}\left(\phi_{Y \backslash c}\right) \rightarrow \mathbf{T}\left(\phi_{Y}\right) \rightarrow 0
\end{aligned}
$$

where the morphism component $\mathbf{T}\left(\phi_{X \cup C}\right) \longrightarrow \mathbf{T}\left(\phi_{X \cup B}\right)$ is 0 if $C \not \subset B$ and otherwise is the canonical inclusion times the sign determined by $\omega$. Then $\mathbf{T}(X, Y, \omega)$ is an acyclic complex.

Proof. We will do induction on $|Y \backslash X|$. If $|Y \backslash X|=0$ then $Y=X$ and $\mathbf{T}(X, Y, \omega)$ : $0 \longrightarrow \mathbf{T}\left(\phi_{Y}\right) \longrightarrow 0$. Suppose now that $X \neq Y$ and let $b$ be the biggest element of $Y \backslash X$. We set $X^{\prime}=X \cup\{b\}, Y^{\prime}=Y \backslash\{b\}$ and let $\omega^{\prime}$ be the induced ordering on $Y^{\prime}$. Then we get the short exact sequence

$$
0 \longrightarrow \mathbf{T}\left(X^{\prime}, Y, \omega^{\prime}\right) \longrightarrow \mathbf{T}(X, Y, \omega) \longrightarrow \mathbf{T}\left(X, Y^{\prime}, \omega^{\prime}\right)\langle-1\rangle \longrightarrow 0 .
$$

Using the induction hypothesis and the long exact sequence in homology we get that if $i>1$ then $\mathrm{H}_{i}(\mathbf{T}(X, Y, \omega))=0$ while if $i=1$ then

$$
0 \longrightarrow \mathrm{H}_{1}(\mathbf{T}(X, Y, \omega)) \longrightarrow \mathrm{H}_{0}\left(\mathbf{T}\left(X, Y^{\prime}, \omega^{\prime}\right)\right) \longrightarrow \mathrm{H}_{0}\left(\mathbf{T}\left(X^{\prime}, Y, \omega\right) .\right.
$$

Thus it suffices to show that the map of complexes

$$
\mathrm{H}_{0}\left(\mathbf{T}\left(X, Y^{\prime}, \omega^{\prime}\right)\right) \longrightarrow \mathrm{H}_{0}\left(\mathbf{T}\left(X^{\prime}, Y, \omega\right)\right)
$$

induced by the inclusion map $\mathbf{T}\left(\phi_{Y^{\prime}}\right) \subset \mathbf{T}\left(\phi_{Y}\right)$ is injective. This however is immediate since

$$
\mathbf{T}_{i}\left(\phi_{Y^{\prime}}\right) \bigcap \bigoplus_{c \in Y \backslash X} \mathbf{T}_{i}\left(\phi_{Y \backslash c}\right)=\bigoplus_{c \in Y^{\prime} \backslash X} \mathbf{T}_{i}\left(\phi_{Y^{\prime} \backslash c}\right),
$$

as follows from the structure of these sets, see [12, Definition 2.4.1].

We apply Lemma 5.4 to the special case where $X=I(\alpha)$ and $Y=I^{\alpha}$. We have that $Y \backslash X=I_{\alpha}$.

Lemma 5.6. Let $\alpha \in \Lambda$ generic, and let $\omega$ be an ordering on $I^{\alpha}$. Then the natural sequence of morphisms of complexes

$$
0 \rightarrow \mathbf{T}\left(\phi_{I(\alpha)}\right) \longrightarrow \bigoplus_{b \in I_{\alpha}} \mathbf{T}\left(\phi_{I(\alpha) \cup\{b\}}\right) \longrightarrow \cdots \bigoplus_{b \in I_{\alpha}} \mathbf{T}\left(\phi_{I^{\alpha} \backslash b}\right) \longrightarrow \mathbf{T}\left(\phi_{I^{\alpha}}\right) \rightarrow 0
$$

is acyclic.

We can now prove the main result of this paper:

Theorem 5.8. Let $\alpha \in \Lambda$ be generic. There is at most one $i \geq 1$ such that $\beta_{i, \alpha}(L) \neq 0$. More precisely, we have for each $i \geq 1$

$$
\beta_{i, \alpha}(L)= \begin{cases}\beta\left(\mathbf{M}_{\alpha}\right) & \text { if } i=\left|I_{\alpha}\right|-\operatorname{rank} \mathbf{M}_{\alpha}+1 ; \\ 0 & \text { otherwise. }\end{cases}
$$


Proof. Let $b \in I_{a}$ and let $\omega$ be an ordering on $I^{\alpha}$ so that $b$ is the biggest element of $I_{\alpha}$. The two standard spectral sequences associated with the double complex of Lemma 5.6 collapse. According to the first filtration we get the complex $V(\alpha, \phi, \omega)$ •, see the remarks preceding Lemma 5.1. According to the second filtration we get the complex $C(\alpha)$. . Thus $\mathrm{H}_{i}(C(\alpha))=\mathrm{H}_{i}(V(\alpha))$. Combining this with Lemma 5.3 and Corollary 4.9 we are done.

We apply the theorem to monomial ideals. When $J$ is a monomial ideal and $\alpha$ is generic we prove that $\beta_{i, \alpha}(R / J) \neq 0$ if and only if $\alpha$ corresponds to a face of the Scarf complex of $R / J$.

Corollary 5.9. Let $J$ be a monomial ideal and let $\alpha \in \Lambda(R / J)$ be a generic element. If $I_{\alpha} \neq I^{\alpha}$ then $\forall i \geq 1, \beta_{i, \alpha}(R / J)=0$. Otherwise

$$
\beta_{i, \alpha}(R / J)= \begin{cases}1 & \text { if } i=\left|I_{\alpha}\right| \\ 0 & \text { otherwise }\end{cases}
$$

Proof. If $I_{\alpha} \neq I^{\alpha}$ then $\mathbf{M}_{\alpha}$ is the empty matroid and $\beta\left(\mathbf{M}_{\alpha}\right)=0$. Otherwise $\beta\left(\mathbf{M}_{\alpha}\right)=\operatorname{rank} \mathbf{M}_{\alpha}=1$.

We finish this section with an example to show that in the general case it may be $I_{\alpha} \neq I^{\alpha}$ and $\beta_{i, \alpha}(L) \neq 0$.

Example 5.10. Let $L$ be the module of Example 4.10. For $\alpha=(3,3,3)$ we have the following data: $\beta\left(\mathbf{M}_{\alpha}\right)=\operatorname{rank} \mathbf{M}_{\alpha}=1,\left|I_{\alpha}\right|=3$. Thus $\beta_{3, \alpha}(L)=1$.

\section{REFERENCES}

[1] D. Bayer, I. Peeva, And B. Sturmfels, Monomial resolutions, Math. Res. Lett. 5 (1998), 31-46.

[2] A. BJöRner, Homotopy type of posets and lattice complementation, J. Combin. Theory Ser. A 30 (1981), 90-100.

[3] A. BJÖRner, The homology and shellability of matroids and geometric lattices, in Matroid Applications, N. White, ed., Cambridge Univ. Press, Cambridge, 1992, pp. 226-283.

[4] A. Björner And J. Walker, A homotopy complementation formula for partially ordered sets, European J. Combin. 4 (1983), 11-19.

[5] A. BJörner ANd G. Ziegler, Broken circuit complexes: factorizations and generalizations, J. Combin. Theory Ser. B 51 (1991), 96-126.

[6] W. Bruns, J. Herzog, On multigraded resolutions, Math. Proc. Cambridge Philos. Soc. 118 (1995), 245-257

[7] H. Charalambous and A. TChernev, Free resolutions for multigraded modules: a generalization of Taylor's construction, Math. Res. Lett. 10 (2003), 535-550.

[8] H. Crapo, A higher invariant for matroids, J. Combin. Theory 2 (1967), 406-417.

[9] E. Miller, B. Sturmfels, Combinatorial Commutative Algebra, Springer (2005).

[10] E. Miller, B. Sturmfels and K. Yanagawa, Generic and Cogeneric Ideals, J. Symb. Computation. 29 (2000), 630-645.

[11] J. OxLeY, Matroid Theory, Oxford Sci. Publ., Oxford University Press, New York, 1992.

[12] A. TChennev, Representations of matroids and free resolutions for multigraded modules, Adv. Math. 208 (2007) no. 1, 75-134.

[13] M. Wachs and J. Walker, On geometric semilattices, Order 2 (1986), 367-385.

[14] D. J. A. Welsh, Matroid Theory, L. M. S. Monographs 8, Academic Press, London-New York, 1976.

[15] G. ZIEGLER, Matroid shellability, $\beta$-systems, and affine hyperplane arrangements, J. Algebraic Combin. 1 (1992), 283-300. 
Department of Mathematics, Aristotle University of Thessaloniki, Greece

E-mail address: hara@math.auth.gr

Department of Mathematics, University at Albany, SUny, Albany, NY 12222

E-mail address: tchernev@math.albany.edu 